\title{
Genomic arrays for the identification of high-risk chronic lymphocytic leukemia: ready for prime time?
}

\author{
Antonio Cuneo, ${ }^{1}$ Gian Matteo Rigolin ${ }^{1}$ and Cristina Mecucci ${ }^{2}$ \\ ${ }^{1}$ Hematology, Department of Medical Sciences, St. Anna University Hospital, Ferrara and ${ }^{2}$ Hematology, Department of Medicine, \\ University of Perugia, Perugia, Italy \\ E-mail: CUNEO ANTONIO - cut@unife.it
}

doi:10.3324/haematol.2020.264689

$\mathrm{H}$ ematopoietic tumors develop through the acquisition of multiple molecular cytogenetic lesions, which confer growth advantage to the neoplastic cells, favoring the emergence of clones resistant to treatment. Not surprisingly, a high number of chromosome aberrations represents an unfavorable prognostic factor in several hematologic neoplasias, including acute myeloid leukemia, ${ }^{1}$ myelodysplastic syndrome, ${ }^{2}$ myelofibrosis ${ }^{3}$ and chronic lymphocytic leukemia (CLL). ${ }^{4}$

Conventional banding analysis (CBA) is a well-established method allowing for a complete overview of the cell genome, with high specificity and low sensitivity. The presence of three or more chromosome lesions in the same clone, usually referred to as complex karyotype, was associated with a shorter overall survival in CLL as early as $1990 .{ }^{5}$ Since then, reports have documented the negative prognostic significance of a complex karyotype in patients treated with chemoimmunotherapy and with novel mechanism-based therapy. ${ }^{6-10}$

While prognostic factors associate with outcome inde- pendently of the treatment, predictive factors are able to identify patients who are likely to obtain improved survival with a specific therapy and can only be identified in comparative trials. ${ }^{11}$ Because different types of treatment can be offered to CLL patients, biological factors predicting outcome with a specific drug are very useful in clinical practice and CBA was proposed as a desirable procedure in the diagnostic workup within prospective clinical studies. ${ }^{12}$ Interestingly, high efficacy of venetoclax plus obinutuzum$\mathrm{ab}$ in patients with a complex karyotype and CLL was recently documented in the CLL14 trial, ${ }^{13}$ pointing to a possible role of complex karyotype as a predictive marker.

Despite the introduction of effective mitogens, CBA is quite a cumbersome procedure requiring fresh dividing cells. For this reason, molecular methods such as array comparative genomic hybridization $(\mathrm{CGH})$, single nucleotide insertional polymorphisms and next-generation sequencing have been applied in CLL, consistently showing that a high number of genetic lesions is associated with an inferior outcome. ${ }^{14-16}$

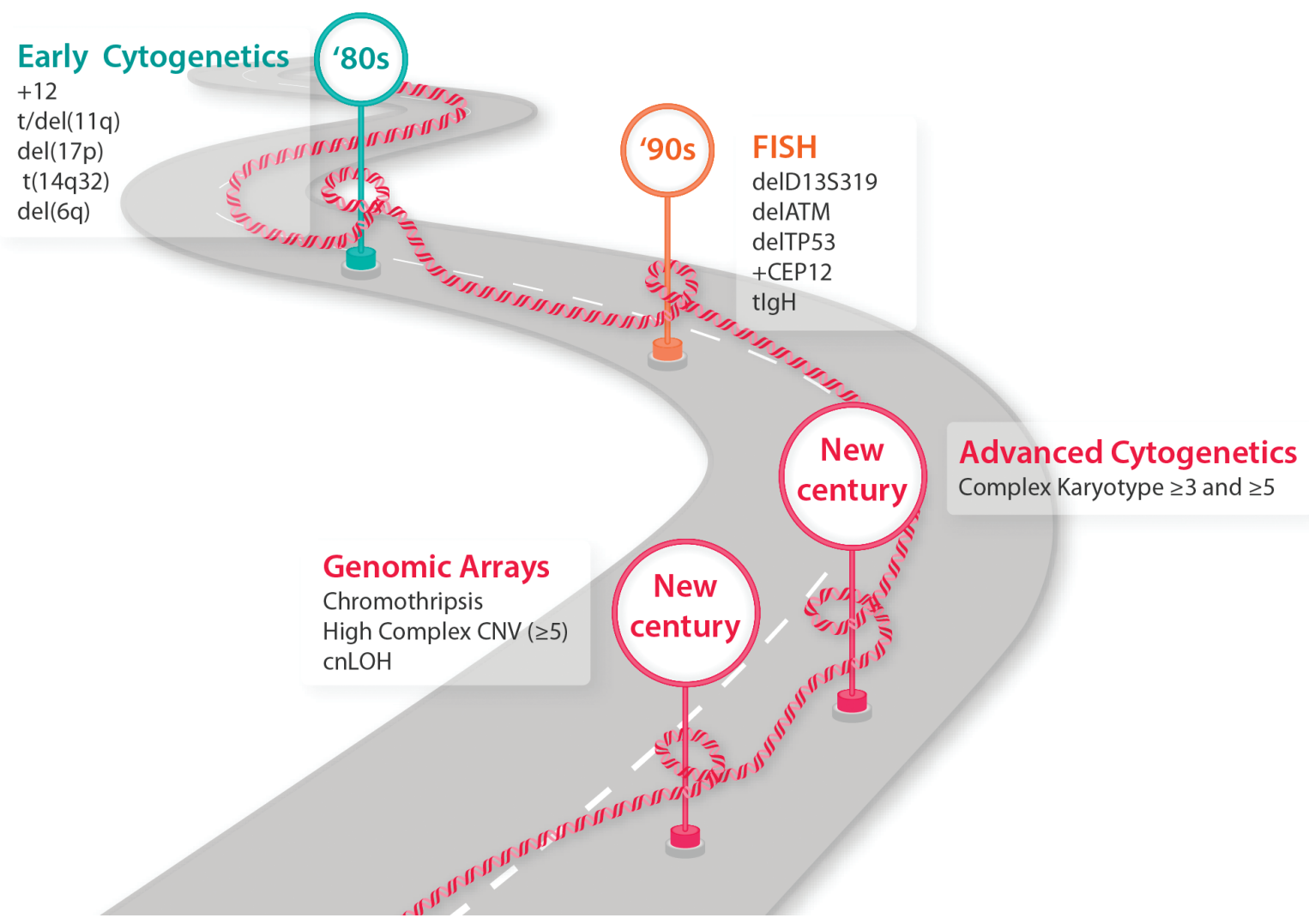

The long road of genetic advances with a clinical impact on chronic lymphocytic leukemia. CNV: copy number variation; cnLOH: copy neutral loss of heterozygosity; $\geq 3$ and $\geq 5$ : three or more, or five or more aberrations in the same clone, respectively. 
In this issue of Haematologica, Leeksma and co-work$\mathrm{ers}^{17}$ report the results of high-resolution genome-wide detection of copy-number alterations (CNA) in a large multicenter cohort of CLL patients, showing that genomic arrays are more sensitive than molecular cytogenetic methods and that high genomic complexity (i.e., 5 or more $\mathrm{CNA}$ ) represents an independent adverse prognosticator.

In their analysis, 13 laboratories connected to the European Research Initiative in CLL (ERIC) group analyzed 2,293 patients by array-CGH and performed concurrent fluorescence in situ hybridization (FISH) in 260 patients using the classical four-probe panel detecting $17 \mathrm{p}-, 11 \mathrm{q}-,+12$ and 13q- and concurrent CBA in 122 patients. Nine-hundred seventy-two patients, known to be untreated at the time of sampling, were included in the overall survival analyses.

Overall, CNA were detected in $78.9 \%$ of the cases and, as expected, previously treated patients and patients with high-risk CLL (i.e., in advanced stage or with unfavorable genetic features) had a significantly higher number of CNA.

In one-third of the cases genomic lesions detected by array-CGH were not detected by FISH. However, despite a high concordance between the two approaches, subclonal chromosome lesions detected by FISH, including $11 \mathrm{q}-$ and $17 \mathrm{p}-$, were not detected by array-CGH.

In previous analyses, similar results were observed when comparing FISH and CBA. ${ }^{18,19}$ Interestingly, Leeksma and co-workers suggest that CGH may be more sensitive than CBA, given that a direct comparison of results from 122 patients showed chromosomal abnormalities in $86.1 \%$ of patients by genomic arrays versus $73.8 \%$ by $\mathrm{CBA}(P=0.015)$.

Among 972 untreated patients, 57 (5.8\%) had high genomic complexity (i.e., $\geq 5 \mathrm{CNA}$ ). This parameter was an independent prognostic factor associated with shorter overall survival (hazard ratio $=2.19 ; 95 \%$ confidence interval: 1.35-3.54) and shorter time to first treatment (hazard ratio $=2.00 ; 95 \%$ confidence interval: 1.28-3.14), thus confirming the results of a recent international report on CBA. ${ }^{8}$ It is worth noting that, as the authors wisely pointed out, this series included patients treated with different chemoimmunotherapy regimens and that the role of genomic complexity in patients treated with novel agents is still unknown.

Besides the classical biomarkers and molecular genetic lesions associated with inferior prognosis, array-CGH identified gains of $8 q$, and losses of $9 p$ and $18 p$ as candidate markers possibly associated with a shorter overall survival in univariable Cox regression analysis. Interestingly, a minority of patients $(\mathrm{n}=32)$ showed very complex rearrangements, consisting of $\geq 10$ oscillating copy number alterations involving two or three copy number states on one chromosome, a pattern suggestive of catastrophic mitotic events, usually referred to as chromothripsis. ${ }^{20}$ Putative chromothripsis was associated with a dismal outcome in this series.

It is worth noting that, despite heterogeneity of the patients and of the methods employed in the different laboratories (i.e., total blood or purified CD19-positive lymphocytes, different array-CGH platforms and proto- cols) the study by Leeksma et al. has the following merits: (i) rigorous criteria were applied for the detection of CNA, with reference to the $5 \mathrm{Mb}$ cut-off that is close to the chromosome band size; (ii) virtually all the results corresponded to established cytogenetic imbalances expected to occur in CLL; and (iii) the highly complex karyotype (i.e., $\geq 5$ changes) was detected, with results in patients being superimposable to those with concurrent CBA.

On the other hand, an intrinsic limit of genomic arrays, like that of other genomic techniques, is the lack of identification of subclones and translocations; furthermore, the major specific contributions generated in this analysis, i.e., chromothripsis and copy neutral loss of heterozygosity, could not be analyzed in detail.

Although not yet validated in prospective studies, genomic arrays could become an accurate tool for CLL risk stratification, as they may represent a widely applicable method for the detection of DNA gains and losses. Indeed, while FISH remains the gold standard for the detection of 11q- and 17p-, the introduction of array$\mathrm{CGH}$ in clinical trials may be advantageous as it may allow for investigations on the role of genomic complexity as a prognostic/predictive factor in clinical trials, assisting clinicians in the choice of the most appropriate treatment for their patients.

\section{Disclosures}

No Conflicts of interest to disclose.

\section{Contributions}

$A C, G M R$ and $C M$ wrote and approved the editorial.

\section{References}

1. Döhner H, Estey E, Grimwade D, et al. Diagnosis and management of AML in adults: 2017 ELN recommendations from an international expert panel. Blood. 2017;129(4):424-447.

2. Greenberg PL, Tuechler H, Schanz J, et al. Revised international prognostic scoring system for myelodysplastic syndromes. Blood. 2012; 120(12):2454-2465.

3. Gangat N, Caramazza D, Vaidya R, et al. DIPSS plus: a refined Dynamic International Prognostic Scoring System for primary myelofibrosis that incorporates prognostic information from karyotype, platelet count, and transfusion status. J Clin Oncol. 2011;29(4):392-397.

4. Rigolin GM, del Giudice I, Formigaro L, et al. Chromosome aberrations detected by conventional karyotyping using novel mitogens in chronic lymphocytic leukemia: clinical and biologic correlations. Genes Chromosomes Cancer. 2015;54(12):818-826.

5. Juliusson G, Oscier DG, Fitchett M, et al. Prognostic subgroups in Bcell chronic lymphocytic leukemia defined by specific chromosomal abnormalities. N Engl J Med. 1990;323(11):720-724.

6. Cavallari M, Cavazzini F, Bardi A, et al. Biological significance and prognostic/predictive impact of complex karyotype in chronic lymphocytic leukemia. Oncotarget. 2018;9(76):34398-34412.

7. Rigolin GM, Saccenti E, Guardalben E, et al. In chronic lymphocytic leukaemia with complex karyotype, major structural abnormalities identify a subset of patients with inferior outcome and distinct biological characteristics. Br J Haematol. 2018;181(2):229-233.

8. Baliakas P, Jeromin S, Iskas M, et al. Cytogenetic complexity in chronic lymphocytic leukemia: definitions, associations, and clinical impact. Blood. 2019;133(11):1205-1216.

9. Thompson PA, O'Brien SM, Wierda WG, et al. Complex karyotype is a stronger predictor than $\operatorname{del}(17 \mathrm{p})$ for an inferior outcome in relapsed or refractory chronic lymphocytic leukemia patients treated with ibrutinib-based regimens. Cancer. 2015;121(20):3612-3621.

10. Anderson MA, Tam C, Lew TE, et al. Clinicopathological features and outcomes of progression of CLL on the BCL2 inhibitor venetoclax. Blood. 2017;129(25):3362-3370 
11. Ballman KV. Biomarker: predictive or prognostic? J Clin Oncol. 2015;33(33):3968-3971.

12. Hallek M, Cheson BD, Catovsky D, et al. IwCLL guidelines for diagnosis, indications for treatment, response assessment, and supportive management of CLL. Blood. 2018;131(25):2745-2760.

13. Al-Sawaf O, Lilienweiss E, Bahlo J, et al. High efficacy of venetoclax plus obinutuzumab in patients with complex karyotype and chronic lymphocytic leukemia. Blood. 2020;135(11):866-870.

14. Delgado J, Salaverria I, Baumann T, et al. Genomic complexity and IGHV mutational status are key predictors of outcome of chronic lymphocytic leukemia patients with TP53 disruption. Haematologica. 2014;99(11): e231-e234.

15. Gunnarsson R, Isaksson A, Mansouri M, et al. Large but not small copy-number alterations correlate to high-risk genomic aberrations and survival in chronic lymphocytic leukemia: a high-resolution genomic screening of newly diagnosed patients. Leukemia. 2010;24(1):211-215.
16. Rigolin GM, Saccenti E, Bassi C, et al. Extensive next-generation sequencing analysis in chronic lymphocytic leukemia at diagnosis: clinical and biological correlations. J Hematol Oncol. 2016;9(1):88.

17. Leeksma AC, Baliakas P, Moysiadis T, et al. Genomic arrays identify high-risk chronic lymphocytic leukemia with genomic complexity: a multicenter study. Haematologica. 2021;106(1):87-97.

18. Haferlach C, Dicker F, Schnittger S, et al. Comprehensive genetic characterization of CLL: a study on 506 cases analysed with chromosome banding analysis, interphase FISH, IgVH status and immunophenotyping. Leukemia. 2007; 21(12):2442-2451.

19. Rigolin GM, Cibien F, Martinelli S, et al. Chromosome aberrations detected by conventional karyotyping using novel mitogens in chronic lymphocytic leukemia with "normal" FISH: correlations with clinicobiologic parameters. Blood. 2012; 119(10):2310-2313.

20. Stephens PJ, Greenman CD, Fu B, et al. Massive genomic rearrangement acquired in a single catastrophic event during cancer development. Cell. 2011;144(1):27-40.

\title{
Targeting the red cell enzyme pyruvate kinase with a small allosteric molecule AG-348 may correct underlying pathology of a glycolytic enzymopathy
}

\author{
Abdu I. Alayash \\ Silver Spring, MD, USA \\ E-mail: ABDU I. ALAYASH - abdu.alayash@fda.hhs.gov
}

Laboratory of Biochemistry and Vascular Biology, Center for Biologics Evaluation and Research, Food and Drug Administration (FDA),

doi:10.3324/haematol.2020.266585

M ature red blood cells (RBC) lack a nucleus and mitochondria, relying almost entirely on their own glycolytic degradative processes to generate energy. One of the major reaction pathways in RBC is the Embden-Meyerhof in which a series of enzymes convert glucose anaerobically into usable energy: adenosine triphosphate (ATP). ${ }^{1}$ The pathway utilizes two ATP to initiate the reaction, with ultimately two more ATP being produced. This pathway is essential to meeting the energy demands of RBC, including maintenance of red cell membrane flexibility and, therefore, impacting RBC shape. ${ }^{1}$

In the Embden-Meyerhof pathway, a pyruvate kinase (PK) isoform unique to $\mathrm{RBC}$, PK-R, is a rate-limiting enzyme that plays a critical role in the formation of pyruvate from phosphoenolpyruvate (PEP) with the simultaneous generation of ATP from adenosine diphosphate $(\mathrm{ADP})^{2}$ (Figure 1). Among the most common enzyme defects related to the Embden-Meyerhof pathway is an inherited disorder in which homozygote individuals display signs and symptoms of hemolytic anemia due to the deficiency of the PK-R enzyme. PK defects have been documented worldwide, although most cases have been identified in people of Northern European ancestry. ${ }^{3}$

This rare hereditary disorder is characterized by changes in RBC metabolism including manifestation of anemia and a compromised energetic profile (ATP production). RBC deficient in PK cannot produce enough energy to maintain normal membrane function. Potassium and water leak from the cell, while calcium concentrations increase. As a result, these cells become rigid, lose flexibility, and are more susceptible to premature hemolysis. ${ }^{4}$

In mammals, two PK genes are expressed depending on anatomical region and cell type: PK muscle (PKM) and PK liver and red blood cell (PKLR). PKLR controls the expres- sion of the red blood cell (PK-R) or liver (PK-L) isoforms from tissue-specific promoters. Mutations in the PKLR gene cause PK deficiency with clinical symptoms apparently confined to RBC. PK-R of RBC is a tetrameric enzyme that exists in equilibrium between a less active T-state and a more active R-state that can be induced by binding to the glycolytic intermediate fructose bisphosphate (FBP). Therefore, intervention strategies designed to counter this condition included the stabilization of the active $\mathrm{R}$ state of PK-R, directly restoring PK activity above and beyond the endogenous activation by FBP. ${ }^{5}$

An early investigation that screened for drugs targeting the PK enzyme resulted in the discovery of a small molecule, AG-348, which allosterically activates wild type PK as well as the mutant form of the enzymes. The activities of this molecule were demonstrated in vitro, in mice, and ex vivo in human RBC. ${ }^{6}$ A partially resolved crystal structure of AG-348 bound to PK-R (2.75 $\mathrm{A}^{\circ}$ resolution) showed that AG-348 is bound in the PK enzyme pocket at the dimerdimer interface away from the FBP-binding domain and is buried in a cluster of apolar amino acids inducing post binding conformational change to the final R-state reminiscent of the classic $\mathrm{R}$ to $\mathrm{T}$ transition in another red cell protein, hemoglobin.

In this issue of Haematologica, Rab et al. ${ }^{8}$ report on the effects of AG-348 on RBC from a small number of patients with PK deficiency. In this investigation, the group carried out ex vivo experiments on RBC from a broad range of patient phenotypes, including measuring several parameters such as activities of the intermediates in the glycolytic pathway and ATP levels. They showed that AG-348 affects thermostability of the PK-R, protein levels and the shape of RBC. They also reported a modest increase in ATP and improvement in PK thermostability. 